\title{
Pathogenesis of Osteosclerotic Change Following Treatment with an Antibody Against RANKL for Giant Cell Tumour of the Bone
}

\author{
MAMER ROSARIO ${ }^{1,2^{*}}$, AKIHIKO TAKEUCHI ${ }^{1 *}$, NORIO YAMAMOTO ${ }^{1}$, KATSUHIRO HAYASHI $^{1}$, \\ SHINJI MIWA ${ }^{1}$, TAKASHI HIGUCHI ${ }^{1}$, KENSAKU ABE $^{1}$, YUTA TANIGUCHI ${ }^{1}$, \\ HISAKI AIBA ${ }^{1}$, YOSHIKAZU TANZAWA ${ }^{3}$, HIDEKI MURAKAMI ${ }^{1}$ and HIROYUKI TSUCHIYA ${ }^{1}$ \\ ${ }^{1}$ Department of Orthopaedic Surgery, Kanazawa University Graduate School of Medical Sciences, \\ Kanazawa, Japan; \\ ${ }^{2}$ Department of Orthopaedics, East Avenue Medical Center, Quezon City, Philippines; \\ ${ }^{3}$ Division of Musculoskeletal Oncology, National Cancer Center Hospital, Tokyo, Japan
}

\begin{abstract}
Background: Giant cell tumours (GCTs) of the bone are intermediate tumours that are locally aggressive. Denosumab, an antibody against receptor activator of nuclear factor kappa-B ligand (RANKL), was recently developed; however, it induces osteosclerotic change through an unknown mechanism. We determined whether osteosclerotic change could be induced by neoplastic stromal cells of giant cell tumours (GCTS). Patients and Methods: Participants included four patients with GCT of the bone who were treated with neoadjuvant denosumab. Expression of alkaline phosphatase (ALP), osteocalcin (OCN), RANKL and histone H3K36 trimethylation were assessed through immunohistochemistry of biopsy and surgical specimens. Results: OCN expression was significantly elevated after denosumab treatment, whereas ALP and RANKL expressions were not significantly elevated. Immunofluorescence staining revealed $O C N$ expression and $H 3 K 36$ trimethylation while their co-localisation was confirmed in the surgical specimens. Conclusion: Denosumab promoted OCN expression and might induce the osteogenic differentiation of GCT stromal cells.
\end{abstract}

*These Authors contributed equally to this study.

Correspondence to: Akihiko Takeuchi, MD, Ph.D., Department of Orthopaedic Surgery, Kanazawa University Graduate School of Medical Sciences, 13-1 Takara-machi, Kanazawa, Ishikawa 9208640, Japan. Tel: +81 762652374, Fax: +81 762344261, e-mail: a_take@med.kanazawa-u.ac.jp

Key Words: Denosumab, giant cell tumour of the bone, giant cell tumour stromal cells, osteosclerotic change, RANKL, alkaline phosphatase, osteocalcin.
Giant cell tumours (GCTs) of the bone are common, primary, intermediate tumours that are locally aggressive (1). Traditionally, surgical tumour removal has been the mainstay of treatment, most commonly by intra-lesional excision with extended curettage and bone cement reconstruction (2); however, adequate tumour resection may not be feasible in the sacrum, spine and base of the skull, while advanced disease may need wide excisions that requires careful preoperative planning to secure margins without damaging nearby neurovascular structures (3).

The discovery that receptor activator of nuclear factor kappa-B ligand (RANKL) was central to the pathogenesis of GCT of the bone has led to the use of the antibody against RANKL, denosumab. Literature reports have noted that new woven bone forms after denosumab treatment in areas that previously had RANKL-positive GCT stromal cells (SCs) (4-6). Several studies have found in vitro evidence that immature osteoblastic phenotypes are maintained among GCT SCs $(7,8)$, and that GCT SCs can differentiate into mature osteoblasts when separated from the osteoclastic component. This allows them to form mature bone when implanted into immunologically deficient mice (9); however, whether the mechanism underlying this new bone formation after denosumab treatment is reactive or neoplastic, requires clarification. Recent reports have identified that driver mutations of glycine 34 in the histone $H 3 F 3 A$ gene are a central feature of GCTs of bone (10 11), and other research has shown that these mutations were associated with H3K36 trimethylation by immunohistochemistry (IHC) (12).

In this study, our aim was to compare the expressions of osteogenic proteins in biopsy and denosumab-treated tumour tissues, and to determine whether the osteosclerotic change was induced by GCT SCs. 
a

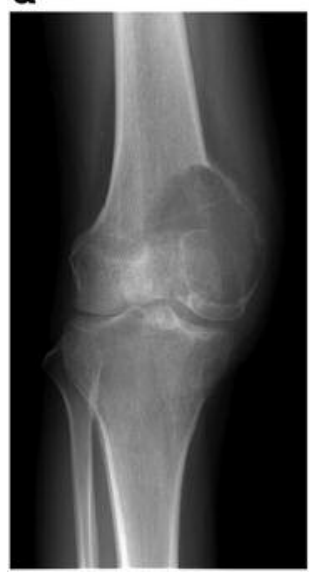

b

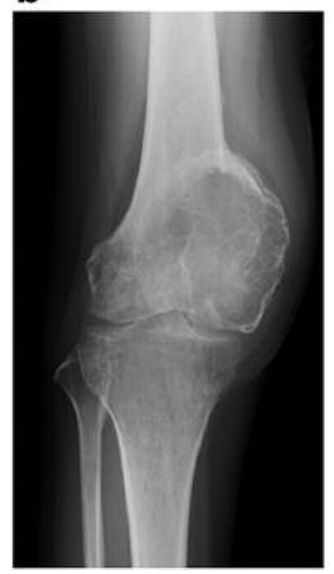

C

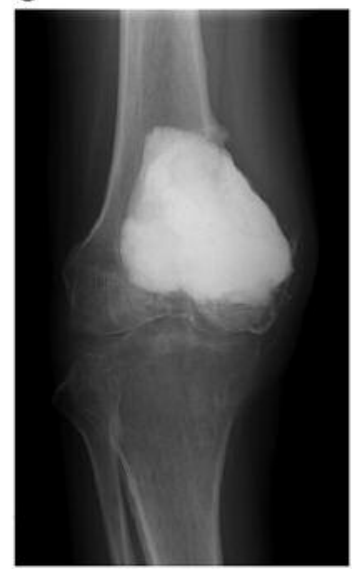

Figure 1. Knee radiograph and treatment course for Case 4. A radiograph taken at presentation revealed an osteolytic lesion in the right distal femur (a); a radiograph after five courses of neoadjuvant denosumab showed signs of new bone formation and reconstituted cortical bone (b); a radiograph taken 6 months after surgery showed stable artificial bone reconstruction without evidence of tumour recurrence (c).

Table I. Patient characteristics.

\begin{tabular}{lcccccc}
\hline Case & Age, years & Gender & Location & Status & Number of denosumab administarations & Surgery \\
\hline 1 & 51 & F & Distal tibia & Recurrent & 12 & Curettage \\
2 & 28 & M & Distal radius & Primary & 6 & Curettage \\
3 & 51 & M & 12th Thoracic vertebra & Primary & 10 & TES \\
4 & 34 & F & Distal femur & Primary & 6 & Curettage \\
\hline
\end{tabular}

TES: Total en bloc spondylectomy.

\section{Patients and Methods}

Study design. We studied four patients with histologically diagnosed GCT of bone who received at least five courses of denosumab (RANMARK $^{\circledR}$; Daiichi Sankyo Co., Ltd., Tokyo, Japan) before surgery. Tissue samples were compared among the patients before and after surgery. The study was approved by the Ethics Committee (No. 2183) for Medical Studies of Kanazawa University Graduate School of Medical Sciences, and written informed consent was obtained from each patient.

Participants. Two men and two women participated, with a mean age of 35 years (range $=31$ to 43 years). The primary lesion sites were of the distal radius, distal femur, 12th thoracic vertebra and distal tibia. All four patients were administered subcutaneous injections of $120 \mathrm{mg}$ denosumab monthly, with additional loading doses on days 8 and 15 . The decision to perform surgery was made after reviewing magnetic resonance and computed tomographic images following the five courses of neoadjuvant denosumab. Three patients underwent intra-lesional curettage (Figure 1), but one underwent total en bloc spondylectomy (Table I) (13).

Tissue analysis. Tissue specimens from each patient taken before and after surgery were fixed in $20 \%$ formalin and embedded in paraffin. For each case, one representative block of formalin-fixed and paraffin-embedded tumour tissue was selected. Immunohistochemical and immunofluorescence analyses were then performed.

Immunohistochemistry. All sections were cut to a thickness of $4 \mu \mathrm{m}$ for IHC. A mouse monoclonal antibody against osteocalcin (OCN) (1:400 dilution; ab13418; Abcam, Cambridge, UK), a rabbit polyclonal antibody against alkaline phosphatase (ALP) (1:50 dilution; ab75699; Abcam) and a rabbit polyclonal antibody against RANKL (1:500 dilution; ab9957; Abcam) were used as primary antibodies. We used anti-mouse or anti-rabbit immunoglobulin $G$ (IgG) conjugated with peroxidase-labelled polymers (EnVision, Dako, Carpinteria, CA, USA) as the secondary antibody. After visualisation of the reaction product, sections were counterstained with Meyer's haematoxylin and cover-slipped for microscopic observation. The appearance of brown stain was considered to indicate immunopositivity. Negative controls were created by excluding the primary antibody.

All positive and negative cells were counted in a minimum of five non-overlapping visual fields at $\times 200$ magnification. The labelling indices for OCN, ALP and RANKL were calculated as the percentage of positive cells among the total number of cells per visual field (14). The labelling index was determined by two assessors (MR and AT) blinded to patient outcome, and the assessment of the labelling index was duplicated. 


\section{a}

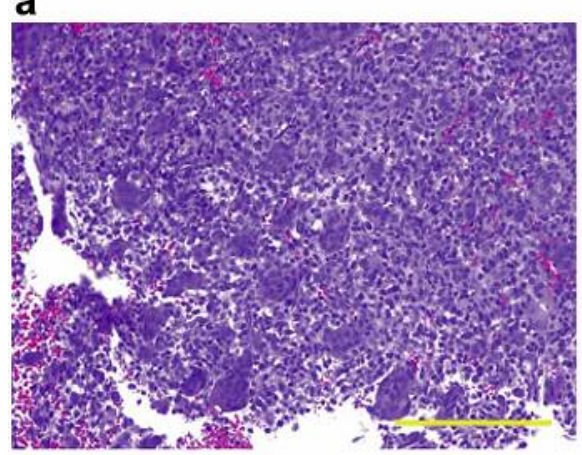

\section{b}

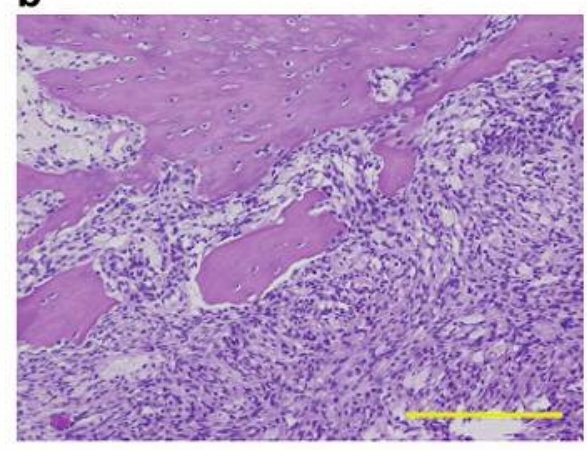

Figure 2. Histological analysis of tumour from Case 4. The biopsy specimen shows numerous mononuclear stromal cells with multinucleated giant cells (a); the post-surgical specimen shows mononuclear stromal cells, new bone formation and loss of multinucleated giant cells (B) (haematoxylin and eosin stain; scale bar $=200 \mu \mathrm{m}$ ).

a

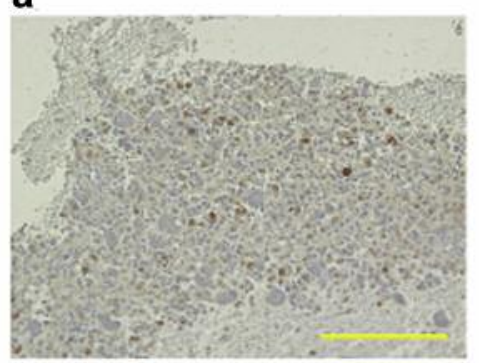

C

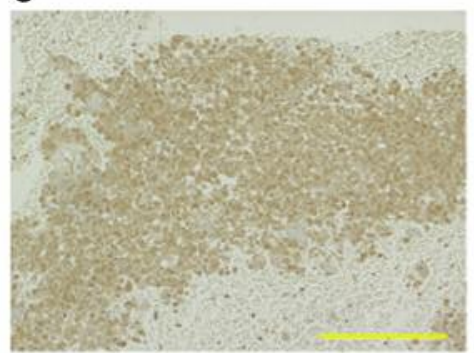

e

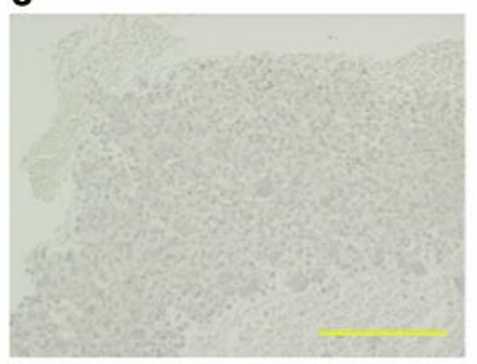

b



d



f



Figure 3. Immunohistochemistry for receptor activator of nuclear factor kappa-B ligand (RANKL), alkaline phosphatase (ALP) and osteocalcin $(O C N)$ in Case 3. There is positive staining among mononuclear cells. RANKL expression in the biopsy (a) and post-surgical (b) specimens was positive. ALP expression in the biopsy (c) specimen was positive, but strong expression was observed in the post-surgical specimen (d). OCN expression was very weak in the biopsy specimen (e), but there was diffuse strong staining in the post-surgical specimen $(f)$. Scale bar=200 $\mu m$.

Immunofluorescence. For immunofluorescence staining, the primary antibodies used were a mouse monoclonal antibody against OCN (1:400 dilution; ab13418; Abcam) and a rabbit polyclonal antibody against trimethylated H3K36 (1:1000 dilution; ab9050; Abcam); the secondary antibodies were rat anti-mouse $\mathrm{IgG}$ fluorescein isothiocyanate (1:400 dilution; eBioscience, San Diego, CA, USA) 
and donkey anti-rabbit IgG (F2 fragments) conjugated to phycoerythrin (1:400 dilution; eBioscience). We performed nuclear staining using 4',6-diamidino-2-phenylindole (Vectashield ${ }^{\circledR}$; Vector Laboratories, Inc., Burlingame, CA, USA), and obtained images using a fluorescence microscope (BZ-9000; Keyence, Osaka, Japan).

Statistical analysis. Mean labelling indices for ALP, OCN and RANKL expression from the pre-surgical tissue specimens were compared with corresponding mean labelling indices from the postsurgical specimens by Mann-Whitney $U$-test. For each test, the result was considered statistically significant when a $p$-value of less than 0.05 was achieved. All statistical analyses were performed using IBM SPSS version 19.0 (IBM Corp., Armonk, NY, USA).

\section{Results}

All cases showed tumour shrinkage and marked new bone formation following denosumab treatment (Figure 1b). Histological analysis revealed decreased numbers of multinucleated giant cells and new woven bone formation (Figure 2). Immunopositivity for RANKL was detected among mononuclear cells in specimens before (Figure 3a) and after (Figure 3b) surgery. ALP expression was detected among mononuclear cells in the biopsy specimens (Figure 3c), but diffuse strong staining was present in the post-surgical specimens (Figure 3d). In contrast, OCN expression was very weak in the biopsy specimens (Figure 3e); however, diffuse strong staining was noted in the post-surgical specimens (Figure 3f).

There was no significant difference between the mean RANKL labelling indices after denosumab treatment and that before $(p=1.000)$ (Figure 4). Although the mean ALP labelling index after denosumab treatment was higher than that before (Figure 4$)$, the difference was not statistically significant $(p=0.149)$. The mean labelling index of the OCN specimens after denosumab treatment was significantly higher than that before $(p=0.029)$ (Figure 4$)$.

H3K36 trimethylation was detected in all post-surgical specimens. Moreover, immunofluorescence double staining for OCN expression (Figure 5a) and trimethylation of H3K36 (Figure 5b) showed their partial co-localization, not only in residual tumour tissue but also in newly formed bone (Figure 5c).

\section{Discussion}

Intra-lesional curettage remains the accepted standard of care for managing GCT of bone surgically (15), even though recurrence rates of $50 \%$ continue to plague this strategy (16). The key to successful therapy for GCT of bone must, therefore, be in ensuring that all margins are clear and all residual microscopic lesions have been eliminated; however, in addition to these baseline concerns about recurrence, it is important to consider that surgery in some locations can entail greater morbidity. Thus, location should be considered as an equally important factor when treating GCT of bone.

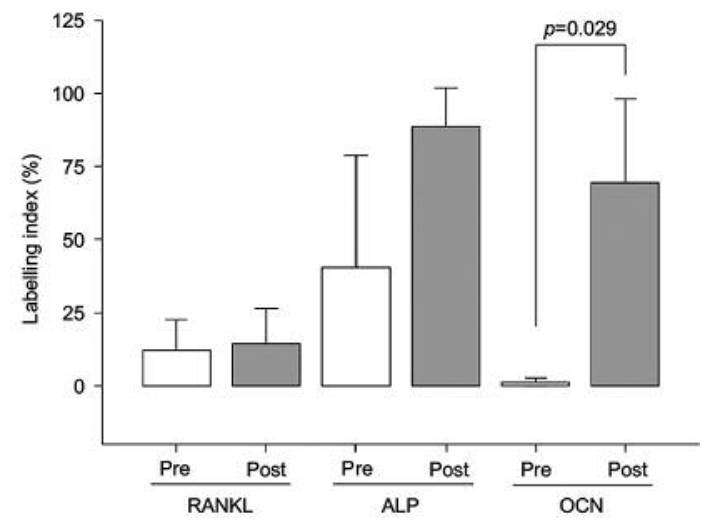

Figure 4. Mean labelling index for receptor activator of nuclear factor kappa-B ligand (RANKL), alkaline phosphatase (ALP) and osteocalcin (OCN) for the denosumab pre-and post-treatment groups. There was no significant difference between the mean RANKL labelling indices pre-and post-treatment $(p=1.000)$. The mean ALP labelling index after denosumab treatment was higher than that before, although the difference was not statistically significant $(p=0.149)$. The mean labelling index for OCN in specimens after denosumab treatment was significantly higher than that before $(p=0.029)$.

In this study, OCN expression was significantly increased following denosumab treatment, and baseline ALP expression was relatively high. Salerno et al. similarly found ALP to be consistently expressed in GCT of bone tissue samples, with GCT SCs showing variable levels of ALP activity (8). We detected RANKL-positive cells in all treated specimens, with the GCT SCs that were positive for H3K36 trimethylation showing OCN expression. Cleven et al. , although acknowledging that $\mathrm{H} 3 \mathrm{~K} 36$ trimethylation was not a surrogate marker for $H 3 F 3 A$ mutation, used IHC analysis to show that increased $\mathrm{H} 3 \mathrm{~K} 36$ trimethylation was associated with mutations in $H 3 F 3 A$ (12). It is difficult to detect $H 3 F 3 A$ mutation status directly by IHC. We identified GCT SCs as those that expressed trimethylated $\mathrm{H} 3 \mathrm{~K} 36$, and colocalization of OCN expression and trimethylated H3K36 in both residual tumour and newly-formed bone tissues suggests that there was osteogenic differentiation of GCT SCs. Denosumab therapy, which is targeted and repeatable (15), therefore, seems promising for both surgical downstaging and eliminating residual microscopic lesions following intra-lesional curettage. It appears to act in this setting by inducing osteogenic differentiation of GCT SCs.

The emphasis on differentiation to mature osteoblasts following denosumab treatment is paramount. Girolami et al. did not find significant modification of runt-related transcription factor 2 (RUNX2) nor of special AT-rich sequence-binding protein 2 (SATB2) expression in GCT of bone samples following denosumab treatment (5). Although both RUNX2 and SATB2 are osteoblast markers, they work synergistically to activate osteogenesis through a regulatory 


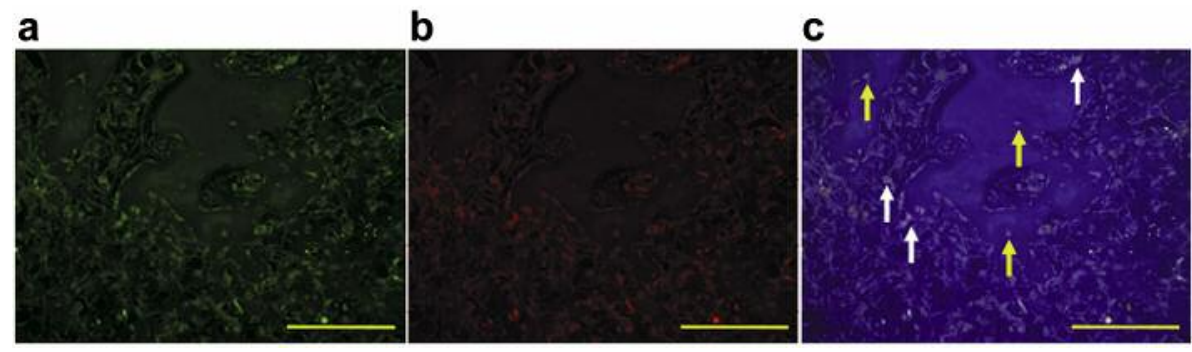

Figures 5. This image shows a representative immunofluorescence result on confocal microscopy for Case 3. The expression patterns for osteocalcin (a) and trimethylation of histone H3K36 (b) were determined and the images then merged with that for 4',6-diamidino-2-phenylindole staining (c). Partial co-localisation of OCN expression and H3K36 trimethylation was observed (white arrows show the co-localisation within residual tumour tissue, while yellow arrows show the co-localisation within newly formed bone). Scale bar=200 $\mu \mathrm{m}$.

circuit with a microRNA cluster (17-19); thus, they are both early, not late, markers of osteogenic differentiation. Nevertheless, the findings of that study support the hypothesis that an immature osteoblastic phenotype is maintained in GCT SCs $(7,8)$.

Osteogenic differentiation is regulated by several key factors at both the gene and protein level, some of which are commonly used differentiation markers. RUNX2 is crucial when generating mineralised tissue, and expression of its gene is usually analysed for assessments during the early phases of osteogenic differentiation $(20,21)$. Another important marker is the effector protein ALP, which is responsible for mineralisation of the extracellular matrix (ECM) (22). Both mRNA and protein expression of ALP can be used to describe the progression of osteogenic differentiation (17). The main constituent of the organic part of the ECM is collagen type I, but it is the two noncollagenous bone ECM proteins, osteopontin and OCN, which are usually used as early and late markers of osteogenic differentiation, respectively (23). Moreover, some studies analysing in vitro osteogenic differentiation in GCT of bone specimens have utilized both ALP and OCN as markers of mature osteoblasts $(24,25)$.

In the study by Branstetter et al. analysing pathological changes in unresectable or recurrent GCT of bone samples from patients treated with denosumab, significant decreases were observed in the numbers of both tumour-associated giant cells and GCT SCs (4). In this study, RANKL-positive cells were found to persist after denosumab treatment. Lau et al. compared the direct effects of both denosumab and zoledronic acid on GCT SCs, and demonstrated reduced cell growth of GCT SCs treated with zoledronic acid, but not of those treated with denosumab (26). Their findings have been supported by recent studies showing the persistence of proliferating GCT SCs despite denosumab treatment $(5,6,27,28)$.

Notably, the inhibitory activity of proinflammatory cytokines on stromal cell differentiation at an early stage $(29,30)$ indicates that we can now link two mechanisms of action observed in denosumab-treated GCT of bone samples: the induction of the RANK-RANKL pathway and that of new bone formation. GCT SCs are capable of differentiating into a mature osteoblast phenotype when separated from the osteoclastic component (9), which is considered a paracrine effect induced by a reduction in tumour necrosis factor-alpha and interleukin-1-beta secretion by giant cells (8). Therefore, denosumab may restore the osteogenic differentiation of GCT SCs by the same paracrine effect that is a result of its inhibition of giant cell differentiation. These findings suggest that surgeons should acknowledge the fact that residual stromal cells may persist in newly formed woven bone and that this persistence may lead to local recurrence.

In conclusion, we showed that denosumab promoted tumour shrinkage, osteosclerotic change and OCN expression. Denosumab might also induce osteogenic differentiation of GCT SCs; however, it is important to remember that we only analysed a small number of patients who required neoadjuvant denosumab treatment at our hospital. Thus, the findings of this study should be considered preliminary. Furthermore, a larger cohort analysis is necessary.

\section{Acknowledgements}

The Authors thank Ms. Yoko Kasai for her assistance.

\section{References}

1 Thanasou NA, Bansal M, Forsyth R, Reid RP and Sapi Z: Giant cell tumour of bone. In: Tumours of Soft Tissue and Bone (Fletcher CDM, Bridge J, Hogendoorn P, Mertens F (eds.). Pathology and Genetics. World Health Organization Classification of Tumours, 4th edn. Lyon, IARC Press, pp. 321-324, 2013.

2 McDonald DJ, Sim FH, McLeod RA and Dahlin DC: Giant-cell tumor of bone. J Bone Joint Surg Am 68: 235-242, 1986.

3 Singh AS, Chawla NS and Chawla SP: Giant-cell tumor of bone: treatment options and role of denosumab. Biologics 9: 69-74, 2015 . 
4 Branstetter DG, Nelson SD, Manivel JC, Blay JY, Chawla S, Thomas DM, Jun S and Jacobs I: Denosumab induces tumor reduction and bone formation in patients with giant-cell tumor of bone. Clin Cancer Res 18: 4415-4424, 2012

5 Girolami I, Mancini I, Simoni A, Baldi GG, Simi L, Campanacci D, Beltrami G, Scoccianti G, D'Arienzo A, Capanna R and Franchi A: Denosumab treated giant cell tumour of bone: a morphological, immunohistochemical and molecular analysis of a series. J Clin Pathol 69: 240-247, 2016.

6 Rekhi B, Verma V, Gulia A, Jambhekar NA, Desai S, Juvekar SL, Bajpai J and Puri A: Clinicopathological features of a series of 27 cases of post-denosumab treated giant cell tumors of bones: a single institutional experience at a tertiary cancer referral centre, India. Pathol Oncol Res 23: 157-164, 2016.

7 Huang L, Teng XY, Cheng YY, Lee KM and Kumta SM: Expression of preosteoblast markers and CBFA-1 and Osterix gene transcripts in stromal tumour cells of giant cell tumour of bone. Bone 34: 393-401, 2004.

8 Salerno M, Avnet S, Alberghini M, Giunti A and Baldini N: Histogenetic characterization of giant cell tumor of bone. Clin Orthop Relat Res 466: 2081-2091, 2008

9 James IE, Dodds RA, Olivera DL, Nuttall ME and Gowen M: Human osteoclastoma-derived stromal cells: correlation of the ability to form mineralized nodules in vitro with formation of bone in vivo. J Bone Miner Res 11: 1453-1460, 1996.

10 Presneau N, Baumhoer D, Behjati S, Pillay N, Tarpey P, Campbell PJ, Jundt G, Hamoudi R, Wedge DC, Loo PV, Hassan AB, Khatri B, Ye H, Tirabosco R, Amary MF and Flanagan AM: Diagnostic value of $H 3 F 3 A$ mutations in giant cell tumour of bone compared to osteoclast-rich mimics. J Pathol Clin Res 1: 113-123, 2015.

11 Behjati S, Tarpey PS, Presneau N, Scheipl S, Pillay N, Van Loo P, Wedge DC, Cooke SL, Gundem G, Davies H, Nik-Zainal S, Martin S, McLaren S, Goody V, Robinson B, Butler A, Teague JW, Halai D, Khatri B, Myklebost O, Baumhoer D, Jundt G, Hamoudi R, Tirabosco R, Amary MF, Futreal PA, Stratton MR, Campbell PJ and Flanagan AM: Distinct $H 3 F 3 A$ and $H 3 F 3 B$ driver mutations define chondroblastoma and giant cell tumor of bone. Nat Genet 45: 1479-1482, 2013.

12 Cleven AH, Hocker S, Briaire-de Bruijn I, Szuhai K, CletonJansen AM and Bovee JV: Mutation analysis of H3F3A and $H 3 F 3 B$ as a diagnostic tool for giant cell tumor of bone and chondroblastoma. Am J Surg Pathol 39: 1576-1583, 2015.

13 Tomita K, Kawahara N, Baba H, Tsuchiya H, Fujita T and Toribatake Y: Total en bloc spondylectomy. A new surgical technique for primary malignant vertebral tumors. Spine (Phila Pa 1976) 22: 324-333, 1997.

14 Scotlandi K, Serra M, Manara MC, Maurici D, Benini S, Nini G, Campanacci M and Baldini N: Clinical relevance of Ki-67 expression in bone tumors. Cancer 75: 806-814, 1995.

15 Yang T, Zheng XF, Li M, Lin X and Lin Q: Stimulation of osteogenic differentiation in stromal cells of giant cell tumor of bone by zoledronic acid. Asian Pac J Cancer Prev 14: 5379$5383,2013$.

16 Sung HW, Kuo DP, Shu WP, Chai YB, Liu CC and Li SM: Giant-cell tumor of bone: analysis of two hundred and eight cases in Chinese patients. J Bone Joint Surg Am 64: 755-761, 1982.
17 Granéli C, Thorfve A, Ruetschi U, Brisby H, Thomsen P, Lindahl A and Karlsson C: Novel markers of osteogenic and adipogenic differentiation of human bone marrow stromal cells identified using a quantitative proteomics approach. Stem Cell Res 12: 153-165, 2014.

18 Hassan MQ, Gordon JA, Beloti MM, Croce CM, van Wijnen AJ, Stein JL, Stein GS and Lian JB: A network connecting RUNX2, SATB2 and the miR-23a 27a 24-2 cluster regulates the osteoblast differentiation program. Proc Natl Acad Sci USA 107: 19879-19884, 2010.

19 Huang W, Yang S, Shao J and Li Y: Signaling and transcriptional regulation in osteoblast commitment and differentiation. Front Biosci 12: 3068-3092, 2013.

20 Deng ZL, Sharff KA, Tang N, Song WX, Luo J, Luo X, Chen J, Bennett E, Reid R, Manning D, Xue A, Montag AG, Luu HH, Haydon RC and He TC: Regulation of osteogenic differentiation during skeletal development. Front Biosci 13: 2001-2021, 2008.

21 Ducy P, Schinke T and Karsenty G: The osteoblast: a sophisticated fibroblast under central surveillance. Science 289: 1501-1504, 2000.

22 Marom R, Shur I, Solomon R and Benayahu D: Characterization of adhesion and differentiation markers of osteogenic marrow stromal cells. J Cell Physiol 202: 41-48, 2004.

23 Aubin JE: Regulation of osteoblast formation and function. Rev Endocr Metab Disord 2: 81-94, 2001.

24 Robinson D, Segal M and Nevo Z: Giant cell tumor of bone the role of fibroblast growth factor 3 positive mesenchymal stem cells in its pathogenesis. Pathobiology 70: 333-342, 2003.

25 Steensma MR, Tyler WK, Shaber AG, Goldring SR, Ross FP, Williams BO, Healey JH and Purdue PE: Targeting the giant cell tumor stromal cell: functional characterization and a novel therapeutic strategy. PLoS One 8: e69101, 2013.

26 Lau CP, Huang L, Wong KC and Kumta SM: Comparison of the anti-tumor effects of denosumab and zoledronic acid on the neoplastic stromal cells of giant cell tumor of bone. Connect Tissue Res 54: 439-449, 2013.

27 Mak IWY, Evaniew N, Popovic S, Tozer R and Ghert M: A translational study of the neoplastic cells of giant cell tumor of bone following neoadjuvant denosumab. J Bone Joint Surg Am 96: e127(1-8), 2014.

28 Mukaihara K, Suehara Y, Kohsaka S, Akaike K, Tanabe Y, Kubota D, Ishii M, Fujimura T, Kazuno S, Okubo T, Takagi T, Yao T, Kaneko K and Saito T: Protein expression profiling of giant cell tumors of bone treated with denosumab. PLoS One 11: e0148401, 2016.

29 Lacey DC, Simmons PJ, Graves SE and Hamilton JA: Proinflammatory cytokines inhibit osteogenic differentiation from stem cells: implications for bone repair during inflammation. Osteoarthr Cartil 17: 735-742, 2009.

30 Gilbert L, He X, Farmer P, Rubin J, Drissi H, van Wijnen AJ, Lian JB, Stein GS and Nanes MS: Expression of the osteoblast differentiation factor RUNX2 (CBFA1/AML3/PEBP2alpha A) is inhibited by tumor necrosis factor-alpha. J Biol Chem 277: 2695-2701, 2002.

Received November 23, 2016

Revised January 13, 2017 Accepted January 18, 2017 